\title{
Statistical Shape Analysis for 3D Facial Images
}

\author{
M. Nakatsu, X.H. Han \\ Graduate School of Inf. Sci. and Eng. Ritsumeikan Univ. \\ Japan
}

\author{
R. Kimura \\ Graduate School of Medicine, University of the Ryukyus \\ Japan
}

\author{
Y.W. Chen \\ Graduate School of Inf. Sci. and Eng., Ritsumeikan Univ. \\ Japan \\ College of Computer Science and Information Technology, Central South Univ. of Forestry and Technology \\ Hunan, China \\ *Corresponding author
}

\begin{abstract}
Recently, the genetic association of human facial morphological variation attracts substantial attention. This study proposes a general framework for analyzing facial morphology variation using scanned 3D landmarks, and explores the phenotype features of facial morphology for identifying population root of Japanese archipelago. After registration for the dense 3D facial points, we investigate both PCA and Mean Hyperplane for exploring the facial morphological variations. Then, in order to reduce the in-population variance of statistical features, we normalize them firstly, and explore the identification of population using the combined phenotype features. Experiments show that our proposed strategy can give promising identification performances between the Mainland Japanese and the Ryukyuan.
\end{abstract}

Keywords-facial morphology; gene; statistical learning; mean hyperplane; normalization

\section{INTRODUCTION}

The residences of Japanese Archipelago mainly include the three human populations; the Ainu, the Mainland Japanese and the Ryukyuan, which can be inferred by genome-wide singlenucleotide polymorphism (SNP) data and characterized by generic base sequences [1],[2]. In the other hand, the genetic association of human facial morphological variation recently becomes a more and more active research field, which aims to quantitatively analyze the possible relation measure between the gene base and a kind of facial morphological variations [3]-[6]. There are a few studies to associate genetic polymorphisms to common facial morphological variations, which generally focused on the analysis of a specific local region of the facial morphology or several landmarks and then only present the limited morphological phenotypes.

This study attempts to explore the discriminated phenotype features of the common facial morphological variations between the Mainland Japanese and the Ryukyuan; the difference of phenotype features between these two populations is prospected to infer different gene base sequences. In order to explore the phenotype features of facial morphology between the Mainland Japanese and the Ryukyuan, we propose a general framework of 3D facial morphological analysis which mainly includes three steps: registration and landmark correspondence, statistical analysis of 3D facial morphological variation, population classification using facial morphological features.

\section{FRAMEWORK OF 3D FACIAL SHAPE ANALYSIS}

Our proposed framework of 3D facial morphological analysis consists of the following six procedures:

1) Data acquisition: a photogrammetric face scanner is applied for achieving 3D dense facial points by scanning the facial surface of the subjects, who are from the population of the mainland Japanese and the Ryukyuan. The scanned data is a group of 3D surface points with three dimensional coordinate $\left[x_{k}, z_{1}\right]$ in each point, which mainly include the facial region.

2) Data trimming and landmark extraction: Beside the facial region, there includes some additional parts such as ear and neck in the scanned data. Therefore, we firstly trim the unnecessary surface points out and only remain the ones in facial region for more effectively analyze the facial morphological variations. With the 3D trimmed surface points, it is difficult to landmark the distinguishable physical points of the facial and thus we transform the 3D surface points into a 2D image with pixel intensity as the point radius in a fixed $\mathrm{z}$ plane, which is called cylinder transformation. With the transformed 2D images, 17 distinguishable landmarks are manually extracted, which can be used for registration between different samples (the following procedure) and as control points for point correspondence.

3) Registration: in order to reduce the variations of translation, scale, and rotation among individuals, we use Generalized Procrustes Analysis (GPA) [7] to align all samples to a reference sample using the manually extracted landmarks.

4) Point correspondence: two strategies: non-rigid point matching based on thin plate spline (TPS) [8] and a simple cylindrical transformation based method, are applied for corresponding points extraction among the individual faces. Though TPS based method can theoretically promise the accurate correspondence via deformation of local region, it is difficult to guarantee the physical point correspondence. Furthermore, this method needs very complicated calculation and thus is time-consuming as shown in previous work [9]. 
Therefore, this study additionally explores a simple and fast point corresponding method based on cylindrical transformation (CyT); with this strategy, we can flexibly select surface point number for efficient post-analysis.

5) Analysis of Facial morphological variations: Beside the popularly used PCA, a discriminated quantitative validation of morphological variation called mean hyperplane (MHP) is proposed, which can marginally maximize the difference between the two faced populations. In addition, we investigate a feature selection approach to achieve the discriminated phenotype feature (morphological variations) following normalization for distinguishing the two populations.

6) Population identification: the wide-used Support Vector Machine(SVM) is applied for population identification with the selected phenotype features as input.

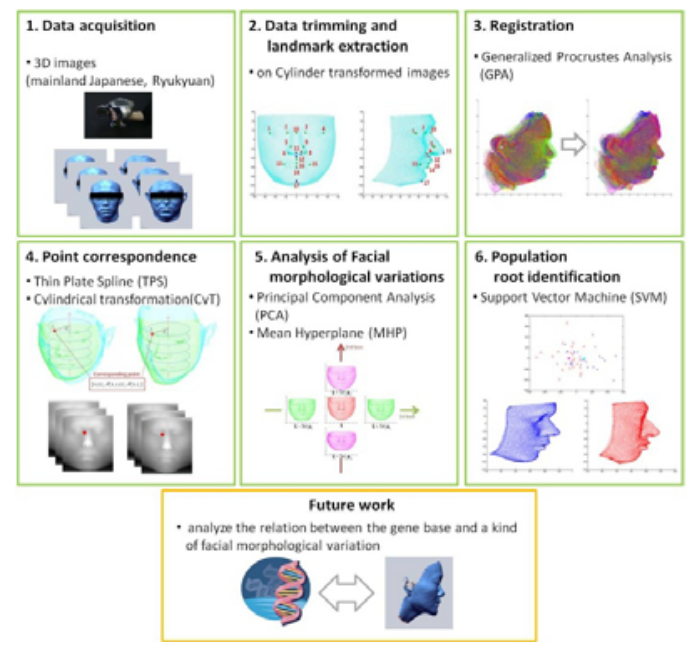

FIGURE I. THE GLOBAL FLOWCHART OF OUR PROPOSAL FRAMEWORK.

\section{ANALYSIS OF FACIAL MORPHOLOGICAL VARIANCE}

The $3 \mathrm{D}$ facial surface points and the obtained 2D images by CyT transformation still have high-dimension, and it is difficult to observe the difference with only a few landmarks between the focused populations, also hard to understand the detail traits of the focused population directly using all the surface points. Thus, this work applies statistical learning methods such as PCA and mean hyperplane for analyzing the facial morphological variations, and use the fisher score for selecting phenotype features to distinguish populations.

\section{A. Morphological Analysis Based on Principal Component} Analysis (PCA)

Principal Component Analysis (PCA) [10] is a widely-used statistical analysis method to de-correlate a multidimensional variable into an orthogonal subspace, which extracts as possible as fewer components to represent the original input space. In our case, PCA projects the high-dimensional facial shape space into a low dimensional subspace which retains as possible as large morphological variations. Then any original facial sample $\mathbf{x}$ can be represented as:

$$
\mathbf{x}=\overline{\mathbf{x}}+b_{1} \mathbf{u}_{1}+b_{2} \mathbf{u}_{2}+\cdots+b_{n k} \mathbf{u}_{w}=\overline{\mathbf{x}}+\sum_{i=1}^{n} b_{l} \mathbf{u}_{l}
$$

where $\mathbf{x}$ denotes the mean vector of training ensemble, $\mathbf{u}_{i}$ is $i^{\text {th }}$ principle component also called basis function extracted by PCA, and $b_{i}$ represents the coefficient, which reflects the possible variation range of the $i^{\text {th }}$ component. It can be seen that any facial morphological sample can be expressed as a linear combination of the mean vector and a group of PCA bases, which are generally sorted or selected according to the variation or the corresponding eigenvalues (called eigenvalue based selection) obtained in PCA procedure. However the selected PCA bases with large morphological variation do not always connect the discriminated phenotype features for distinguishing the two focused populations. Fig. 2 gives the possible morphological variations by the selected PCA bases corresponding to the first two largest eigenvalues. The center facial shape is the mean one of all available training samples, and horizontal and vertical axis represents the possible morphological variations of the 1st and 2nd components, respectively. The left/right and bottom/top facial shapes in Fig.2 range from the combined subtracted/added shapes between the mean one and the $3 \sigma_{1} / \sigma_{2}$ multiplied basis, where above $99 \%$ samples are located in these ranges. The profile views of the morphological variations from the first two selected bases are also given in the top rows, from which we can observe that these mainly manifest the morphological variations of facial length and thickness. In our study, the populations of the mainland Japanese and the Ryukyuan are as the research subjects. As pointed out by anthropology research the facial shape of the mainland Japanese are inclined to be round or oval, and has flat characteristic and slim cheeks, otherwise the Ryukyuan is square, sculptured and of prominent cheeks. Accordingly, the selected PCA bases mainly manifest unrelated variations mentioned by anthropology research between the two investigated populations. Therefore, we aim to explore the discriminated phenotype features for distinguishing the two populations in the following.

Fisher score based basis selection: The key idea of fisher score is to find a subset of features, such that in the subspace spanned by the selected features, the distances between data points in different classes are as large as possible, while the ones between data points in the same class are as small as possible. In this study, we aim to select the discriminated morphological variation analyzed by PCA, which is prospected to give the discriminated phenotype features for identifying the populations of the mainland Japanese and the Ryukyuan. After computing the Fisher score for each basis, we can select the ones with larger fisher score than a predefined threshold or the top L ranked features with large scores. Furthermore, we visualize the morphological variation of the selected first base via Fisher score in the bottom rows of Fig. 3 ; we can observe the concave/convex check variations and the deep/flat eye variations, which are related the opinion of the anthropology researcher. 


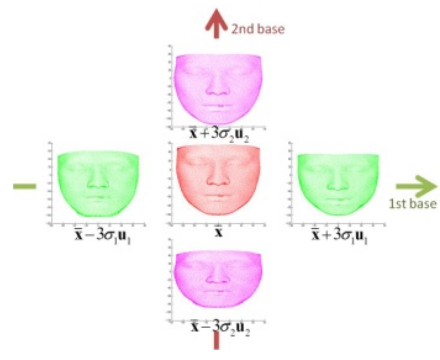

FIGURE II. FACIAL MORPHOLOGY VARIATION .

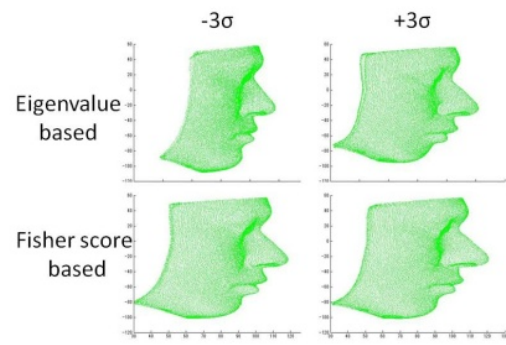

FIGURE III.

COMPARE OF FACIAL MORPHOLOGY VARIATION.

\section{B. Morphological Analysis Based on Mean Hyperplane}

Although the general PCA method can manifest the morphological variations, and the selected PCA bases via fisher score can signify the promising insight from the anthropology researcher. We are more and more interested in the directly variations of statistical difference between the explored populations, which we call as mean hyperplane [11]. The mean hyperplane is simply the normalized difference between the mean vectors of the mainland Japanese and Ryukyuan samples, which can be considered as a basis to represent the morphological variations as illustrated in Fig. 4. In order to calculate the quantitative measure (like the corresponding eigenvalue of each PCA basis) of the variations hold in the basis, we project all facial shape samples on it to produce the coefficient set, and calculate the standard variation, which has the similar meaning to the eigenvalue of a PCA basis. The projected coefficients to the mean hyperplane and its expressed morphological variations are shown in Fig. 4, which manifest clear variation around eye and cheek.

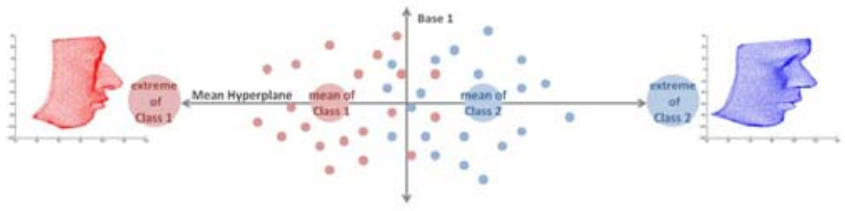

FIGURE IV. FACIAL MORPHOLOGY VARIATION OF MEAN HYPERPLANE.

\section{Normalization of the Selected Phenotype Features}

As introduced in the above subsection, the coefficients of all samples on a basis extracted by PCA or mean hyperplane can be considered as the available samples of a variable. The variables from different selected bases of PCA and mean hyperplane have different variations (the corresponding eigenvalues in PCA and the calculated one from the projected coefficients in mean hyperplane), which will lead to the biased quantitative measure if combining the projected coefficients of different bases for representing a facial sample. Thus, in order to reduce the possible unbalance weights to the coefficients from different bases, we propose two normalization strategies. One is to directly scale the $i$ th coefficient vector $\mathbf{b}_{i}$, which is the concatenated coefficients on the $i$ th basis from all samples, to the range $[0,1]$. The other one is to normalize the coefficient vector using its variance $\lambda$.

After normalization, we concatenate the projected coefficients from all selected bases to represent a facial sample, which is treated as the phenotype features for identifying populations.

\section{EXPERIMENT}

In order to validate the effectiveness of the selected phenotype features, we perform experiments of identification on our prepared dataset, which consists of 3D scanned facial images of 100 mainland Japanese and 100 Ryukyuan that has already done point correspondence. We validate the effectiveness of the proposed mean hyperplane (denoted as MHP) and two normalization strategies (denote norm1 for directly scaling and norm2 for variation based). Fig.5 shows the comparison of MHP and PCA morphological variations and with eigenvalue or fisher based component selection, respectively, MHP shows high accuracies on low feature numbers. Fig. 6 shows the achieved highest accuracies with feature selection strategies.

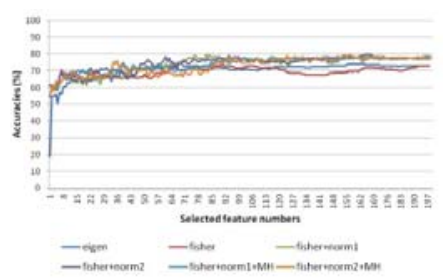

FIGURE V. COMPARED IDENTIFICATION ACCURACIES

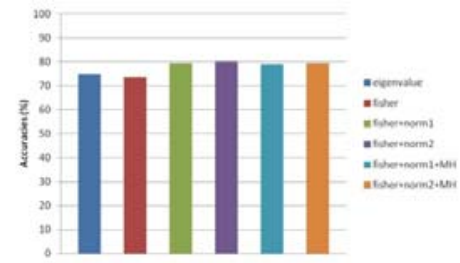

FIGURE VI.

COMPREHENSIVE COMPARISON ON POPULATION IDENTIFICATION.

\section{CONCLUSION}

This study proposed a general framework for analyzing common facial morphological variations, and explored the phenotype features for identifying population of the mainland Japanese and the Ryukyuan. The dense points obtained by a photogrammetric face scanner are corresponded based on TPS-based non-rigid transformation and a cylindrical transformation, and then follow the morphological variation analysis using the statistical learning: PCA and MHP. Furthermore, we probe two morphological base selections to seek more discriminated phenotype features for population identification. Experiments showed that our proposed 
framework with the combined strategies: fisher score based selection of PCA bases, MHP and variation-based normalization achieved the best performance for identifying the mainland Japanese and the Ryukyuan.

This work is supported in part by the MEXT Support Program for the Strategic Research Foundation at Private Universities (2013-2017).

\section{REFERENCES}

[1] Kimura R, Ohashi J, ”Identifying Regions of the Human Genome that Exhibit Evidence for Positive Selection”, eLoS , 2008.

[2] Yamaguchi-Kabata, Y., Nakazono, K., Takahashi, A., Saito, S., Hosono, N., et al., "Japanese population structure, based on SNP genotypes from 7003 individuals compared to other ethnic groups: effects on populationbased association studies", The American J. of Human Genetics, Vol. 83, No. 4, pp.445-456, 2008.

[3] Liu F, van der Lijn F, Schurmann C, Zhu G, Chakravarty MM, et al., ”A Genome-Wide Association Study Identifies Five Loci Influencing Facial Morphology in Europeans.”, PLoS Genetics 8: e1002932 , 2012.

[4] Abzhanov A, Kuo WP, Hartmann C, Grant BR, Grant PR, et al., ”The calmodulin pathway and evolution of elongated beak morphology in Darwin's finches”, Nature 442: pp.563-567 , 2012.

[5] Hubbe M, Hanihara T, Harvati K, "Climate signatures in the morphological differentiation of worldwide modern human populations", Anatomical record (Hoboken, NJ: 2007) 292: pp.1720-1733, 2007.

[6] Harvati K, Weaver TD, "Human cranial anatomy and the differential preservation of population history and climate signatures", The anatomical record Part A, Discoveries in molecular, cellular, and evolutionary biology, 288, pp.1225-1233, 2006.

[7] GRUEN, A. W., and AKCA, M. D., "Generalized procrustes analysis and its applications in photogrammetry", Swiss Federal Institute of Tech., Vol.1, 2003.

[8] Bookstein, F. L., "Principal warps: Thin-plate splines and the decomposition of deformations", Pattern Analysis and Machine Intelligence, Vol.11, No.6, pp.567-585, 1989.

[9] Daisuke Kitabayashi, Guifange Duan, Masataka Seo, Tomoko Tateyama, Eri Miyazato, Kyoko Yamaguchi, Ryosuke Kimura, Xiong Wei, YenWei Chen, "A framework of 3D facial morphology analysis for a genetic association study"[in Japanese], Journal of Japanese Academy of Facial Studies, Vol.13, No.1, pp.63-74, 2013.

[10] Stegmann, Mikkel B., and David Delgado Gomez., ”A brief introduction to statistical shape analysis.", Informatics and Mathematical Modelling Technical University of Denmark, DTU 15, 2002.

[11] Hammond, P., Hutton, T. J., Allanson, J. E., Campbell, L. E, Hennekam, R. C., et al., "3D analysis of facial morphology”, American J. of Medical Genetics Part A, Vol. 126, No.4, pp.339-348, 2004. 